\title{
IV. „Cannae am Don“249. Der Untergang der 8. italienischen Armee im Winter 1942/43
}

\section{Die 8. italienische Armee im Rabmen der Operation "Blau“}

Die ARMIR war im Rahmen der großen deutschen Sommeroffensive an den Don beordert worden. Diese Offensive, die man auch als Hitlers „Zweiten Feldzug“ gegen die Sowjetunion bezeichnet hat ${ }^{250}$, beruhte im wesentlichen auf der Weisung Nr. 41 des "Führers" und obersten Befehlshabers der Wehrmacht vom 5. April 1942, in der die Operationsführung für die kommenden Monate festgelegt wurde. Da die Wehrmacht nicht mehr in der Lage war, wie noch ein Jahr zuvor an allen Frontabschnitten gleichzeitig anzugreifen, sollte der entscheidende Schlag zu dem Zweck, "die den Sowjets noch verbliebene lebendige Wehrkraft endgültig zu vernichten und ihnen die wichtigsten kriegswirtschaftlichen Kraftquellen so weit als möglich zu entziehen “, im Süden der UdSSR geführt werden ${ }^{251}$. Im einzelnen waren mehrere aufeinander folgende, räumlich und zeitlich abgestimmte Teiloffensiven vorgesehen, deren Endziel in der Kaukasusregion lag, die wegen ihrer strategischen Lage als Tor zum Mittleren Osten und wegen ihrer reichen Erdölvorkommen von besonderer Bedeutung war. Auch der Einsatz der verbündeten Armeen - zur Wahrung des nationalen Prestiges in möglichst geschlossenen Formationen mit deutschen ${ }_{n}$ Korsettstangen ${ }^{\alpha 252}$ - wurde in Weisung Nr. 41 in den Grundzügen geregelt:

"Zur Besetzung der sich im Laufe dieser Operation mehr und mehr verlängernden Donfront werden in erster Linie die Verbände der Verbündeten mit der Maßgabe herangezogen, daß deutsche Truppen als starke Stütze zwischen Orel und dem Don sowie an der Stalingrader Landenge einzusetzen sind, im übrigen aber einzelne deutsche Divisionen hinter der Donfront als Eingreifreserven verfügbar bleiben. Die verbündeten Truppen sind weitgehend in eigenen Abschnitten so zu verwenden, daß am weitesten nördlich die Ungarn, demnächst die Italiener, am weitesten südostwärts die Rumänen eingesetzt werden."

Der deutsche Großangriff begann am 28. Juni 1942 und machte zunächst gute Fortschritte; der Vorstoß an den Don gelang ebenso wie die Einnahme von Woronesch. Dagegen scheiterten trotz beachtlichen Geländegewinns alle im Juli unternommenen Versuche, die Masse der gegnerischen Streitkräfte einzuschließen. In dieser Situation entschloß sich Hitler, den ursprünglichen Operationsplan entscheidend abzuändern. Die Heeresgruppe Süd wurde in zwei Heeresgruppen (A und B) aufgespalten, die den Auftrag erhielten, die Offensiven gegen Stalingrad und den Kaukasus nicht mehr nacheinander, sondern gleichzeitig vorzutragen. Daß beide Heeres-

249 Gosztony, Hitlers fremde Heere, S. 299.

250 Wegner, Krieg gegen die Sowjetunion, in: DRZW 6, S. 761; zur militärischen Konzeption und den strategischen Grundlagen der Offensive vgl. ebenda, S. 761-815; ein kurzer Uberblick über Anlage und Verlauf der Operation „Blau“ findet sich bei Kehrig, Stalingrad, S. 25-35.

251 Abgedruckt in: Hitlers Weisungen für die Kriegführung, S. 183-188; die Zitate finden sich auf S. 184 und S. 187.

$252 \mathrm{Vgl}$. hierzu auch den im Detail korrekturbedürftigen, die großen Linien aber überzeugend nachzeichnenden Aufsatz von Hillgruber, Einbau der verbündeten Armeen, hier vor allem S. 670-674. 
gruppen dafür offensichtlich zu schwach waren, störte den Diktator wenig. Daran änderten auch die Einwände von Generalstabschef Halder nichts, der dafür eintrat, zunächst wie vorgesehen gegen Stalingrad zu operieren und erst dann in den Kaukasus vorzustoßen, wenn für den Flankenschutz der angreifenden Verbände gesorgt sei. „Die trichterförmige Ausweitung der Front nach Osten und Süden mußte nach Auffassung des $\mathrm{OKH}$ angesichts des Umfangs der verfügbaren Kräfte zu einer Überdehnung der Frontlinien und zu einer Überforderung der nun zur Sicherung der Don-Stellung eingesetzten verbündeten Armeen führen “253. Dies sei jedoch nur unter der wenig wahrscheinlichen Voraussetzung zu verantworten, daß keine größeren Feindangriffe erfolgten. Doch Hitler ließ sich nicht umstimmen, so daß sich die ursprünglich für einen Nebenkriegsschauplatz vorgesehenen Armeen Italiens, Ungarns und Rumäniens in einem Sektor wiederfanden, der für eine Gegenoffensive der sowjetischen Streitkräfte geradezu prädestiniert war.

Weder Mussolini und das Comando Supremo noch die Führung der 8. Armee waren mit der Aufgabe glücklich, die ihren Verbänden zugewiesen worden war; ein prestigeträchtigerer Kampfauftrag als der, die linke Flanke der auf Stalingrad vorstoßenden Divisionen der 6. Armee und der 4. Panzerarmee zu decken, wäre ihnen sichtlich lieber gewesen ${ }^{254}$. Statt dessen mußte Generaloberst Gariboldi am 13. August die Verantwortung für einen etwa $270 \mathrm{~km}$ breiten Frontabschnitt am mittleren Don zwischen Pawlowsk und der Mündung des Flusses Choper übernehmen, wobei die Soldaten des II. und des XXXV. Armeekorps bis zum 16. August Stellungen bezogen, die bisher von deutschen Einheiten verteidigt worden waren. Es zeigte sich rasch, daß die Rote Armee nicht geneigt war, die Italiener in Ruhe zu lassen. Die Offensive gegen den Sektor der Division „Sforzesca“ Ende August und Angriffe im Bereich des II. Armeekorps im September waren ein deutlicher Beleg hierfür. Daher drängte das Oberkommando der 8. Armee wiederholt sowohl auf die Zuführung deutscher Reserven als auch auf die Verkürzung des zugewiesenen Verteidigungsabschnitts. Die Möglichkeit dazu schien sich im Oktober zu ergeben, als die 3. rumänische Armee zwischen der ARMIR und der 6. deutschen Armee eingeschoben wurde. Doch da sich die Rumänen erfolgreich weigerten, die Ablösung zweier italienischer Divisionen wie befohlen zu vollziehen ${ }^{25}$, blieb es bei einer Frontverkürzung um $40 \mathrm{~km}$, so daß die Verbände des königlichen Heeres noch immer einen Abschnitt von $230 \mathrm{~km}$ Länge zu verteidigen hatten.

Anfang November waren die Umgruppierungen weitgehend abgeschlossen, und die Divisionen hatten ihre Winterstellungen bezogen. Auf dem linken Flügel der 8. Armee stand das Alpinikorps mit den Divisionen "Tridentina“, ${ }_{\text {"Julia }}$ und ${ }_{n} \mathrm{Cu}$ neense", das nördlich von Pawlowsk den Anschluß an die 2. ungarische Armee hielt. Die Alpini waren zunächst im Rahmen der Heeresgruppe A für einen Einsatz im Kaukasus vorgesehen gewesen, dann aber sehr zur Freude von Generaloberst Gariboldi schon am 19. August umdirigiert worden, um die Donfront zu verstär-

253 Wegner, Krieg gegen die Sowjetunion, in: DRZW 6, S. 892.

254 Vgl. Cavallero, Diario, S. 450, Eintrag vom 4. 8. 1942; AUSSME, DS II 1551/1, Aufzeichnungen von Generaloberst Italo Gariboldi über die 8. Armee im Krieg gegen die Sowjetunion vom Dezember 1946, S. 15.

255 Vgl. Kehrig, Stalingrad, S. 55-58, und Wegner, Krieg gegen die Sowjetunion, in: DRZW 6, S. $1008 \mathrm{f}$. 
ken 256. Das II. Armeekorps besetzte mit den Divisionen "Cosseria“ und "Ravenna“ den Abschnitt zwischen Nowaja Kalitwa und Kuselkin, der besonders gefährdet war, weil die Sowjets hier in der Fluß-Schleife von Werch. Mamon einen Brückenkopf besaßen. Rechts davon stand das XXXV. Armeekorps mit der Division „Pasubio" und der 298. deutschen Infanteriedivision, während das XXIX. deutsche Armeekorps mit der Division "Torino“, der 62. deutschen Infanteriedivision und der angeschlagenen Division "Sforzesca" den rechten Flügel der ARMIR bildete und Kontakt mit der 3. rumänischen Armee hielt. An Reserven verfügte die 8. Armee über die 294. Infanteriedivision und die 22. Panzerdivision der deutschen Wehrmacht sowie über die 3. schnelle Division, die jedoch noch von den schweren Kämpfen gezeichnet war, die sie im Juli und August zu bestehen gehabt hatte ${ }^{257}$.

Damit hatte das deutsche Oberkommando eine beachtliche Zahl an eigenen Verbänden im Sektor der 8 . Armee disloziert. Dies kam freilich nicht von ungefähr, da Hitler diesen Abschnitt als besonders gefährdet ansah und eine nördlich von Serafimowitsch angesetzte sowjetische Offensive gegen Rostow für möglich hielt, der die italienischen Truppen ohne Unterstützung nicht gewachsen seien. So hatte er seit dem 16. August 1942 wiederholt darauf gedrängt, deutsche Divisionen zur ARMIR abzustellen, zumal der Angriff der Roten Armee auf die Stellungen der "Sforzesca" seine Befürchtungen zu bestätigen schien. Anfang September spielte er sogar mit dem Gedanken, Verbände aus dem Raum Stalingrad abzuziehen und zur Verstärkung der Donfront einzusetzen ${ }^{258}$. Allerdings konnte sich die 8. Armee nur so lange der Aufmerksamkeit des sprunghaften "Führers" und obersten Befehlshabers der Wehrmacht gewiß sein, wie sein Interesse nicht von den Ereignissen in anderen Sektoren okkupiert wurde. Tatsächlich traten die Geschehnisse an der Donfront nur allzu bald hinter die Schlacht um Stalingrad zurück, ja der Gedanke an eine rasche Eroberung der Stadt entwickelte sich zu einer wahren Obsession, so daß Hitler am 6. Oktober die vollständige Inbesitznahme der Wolgametropole zur wichtigsten Aufgabe der Heeresgruppe B erklärte ${ }^{259}$.

Letztlich wurde in Stalingrad aber nicht nur das Schicksal der dort kämpfenden deutschen Divisionen, sondern auch das Schicksal der ARMIR entschieden. Denn nur bei einem erfolgreichen Abschluß der Operation würden die norwendigen Kräfte freigemacht werden können, um den italienischen Sektor dauerhaft und entscheidend zu verstärken. Der Oberbefehlshaber der Heeresgruppe B, Maximilian Freiherr von Weichs, dem sowohl die 6. deutsche Armee als auch die 8. italienische Armee unterstanden, hatte schon am 24. August offen auf diesen Zusammenhang hingewiesen, als er an Generaloberst Gariboldi schrieb260: „Ich darf erneut versichern, daß nach Abschluß der Kampfhandlungen um Stalingrad der Verteidigungsabschnitt der 8. ital. Armee verkleinert bzw. der Armee ausreichende weitere Kräfte zugeführt werden." Doch Stalingrad fiel nicht, und die Schlacht um die Stadt ver-

256 Vgl. hierzu ausführlich Massignani, Alpini e Tedeschi, S. 105-108.

257 Zur Aufstellung der 8. Armee Anfang November 1942 vgl. Operazioni delle unità italiane al fronte russo, Abbildung 33.

258 Vgl. KTB OKW, Bd. II/1, S. 597, S. 622, S. 642, S. 646 und S. 703, Einträge vom 16. 8., 22. 8., 26. 8., 27. 8. und 9. 9. 1942; Halder, Kriegstagebuch, Bd. 3, S. 523, Eintrag vom 16. 9. 1942. 259 Vgl. Wegner, Krieg gegen die Sowjetunion, in: DRZW 6, S. 987.

260 AUSSME, DS II 1551/1, Maximilian Freiherr von Weichs (Ia Nr. 2633/42 geh. Kdos.) an Italo Gariboldi vom 24. 8. 1942. 
zehrte die deutschen Kräfte schließlich so sehr, daß „der Führung keine andere Wahl mehr [blieb], als Löcher durch das Reißen neuer Löcher zu stopfen “261. Die italienischen Verbände bekamen die Auswirkungen dieses Dilemmas schmerzlich zu spüren. Es fehlte an Transportraum ebenso wie an Treibstoff, es fehlte aber auch an Material zum Ausbau der Stellungen. So kam es im Herbst und Winter 1942 immer wieder zu regelrechten Verteilungskämpfen, die das Verhältnis zwischen Deutschen und Italienern nachhaltig belasteten ${ }^{262}$.

Doch nicht nur die schwierige logistische Situation, die durch den Hang der Intendenza, Nachschubgüter in der Etappe zu horten, anstatt sie an die Truppe auszugeben, zusätzlich verschärft wurde ${ }^{263}$, gab zu Meinungsverschiedenheiten Anlaß. Auch die Organisation der Verteidigung war zwischen italienischen und deutschen Führungsstäben umstritten. Nach den Weisungen, die das Oberkommando der Heeresgruppe B und Hitler selbst erlassen hatten, sollte der Don so nahe am Ufer wie möglich verteidigt werden, wobei der Aufbau einer robusten, durchlaufenden Hauptkampllinie vorgesehen war, auf die vor allem Hitler Wert legte; eine elastische Kampfführung wurde ausgeschlossen 264 . Diese Prinzipien liefen den Auffassungen der italienischen Kommandeure vor allem aus drei Gründen zuwider: Zum einen hätten sie gerne das Gelände besser ausgenutzt, auch um den Preis, die Stellungen weiter vom Flußufer entfernt anzulegen, zum zweiten konnten sie sich nicht an den Gedanken gewöhnen, zugunsten der ersten Verteidigungslinie auf möglichst starke Reserven zu verzichten, und zum dritten hielten sie es für unmöglich, eine durchgehende Hauptkampflinie aufzubauen. Statt dessen ließen sie eine Vielzahl von Stützpunkten anlegen, die meist von einem Zug Infanterie unter dem Befehl eines Leutnants besetzt und durch schwere Waffen wie Granatwerfer und Panzerabwehrkanonen verstärkt wurden. Die deutsche Führung und ihre Verbindungsoffiziere konnten in den ersten beiden Streitfällen Punktsiege davontragen, was das Stützpunktsystem anging, ließen sich die italienischen Stäbe jedoch nicht beirren. Die deutschen Einwände, ein solches Verteidigungssystem sei leicht zu infiltrieren und durch das Herausbrechen einzelner Stützpunkte entscheidend zu schwächen, wurden mit dem Argument gekontert, man habe weder genug Männer noch verläßliche Unterführer in ausreichender Zahl265, um eine Verteidigungsstellung aufzubauen,

261 Wegner, Krieg gegen die Sowjetunion, in: DRZW 6, S. 987.

262 Vgl. Dok. 16, S. 160 ff.; BA-MA, MFB4 18276, Bl. 387-391, Deutscher General beim italienischen AOK 8 (Nr. 902/42 geh.) vom 7. 12. 1942: Besondere Führungsanordnungen für DVSt und DVK Nr. 5; zu den Auseinandersetzungen vgl. z. B. MFB4 41403, Bl. 1025 und Bl. 1033, KTB des Deutschen Generals beim italienischen AOK 8, Einträge vom 2. 9. und 23. 9.1942.

$263 \mathrm{Vgl}$. Rochat, Truppe italiane, S. 40.

264 Vgl. u. a. Instruktionen zur Verteidigung von Wolga und Don, gez. Maximilian Freiherr von Weichs, abgeduckt in: Operazioni delle unità italiane al fronte russo, S. 654 ff.; "Führerbefehl“ ${ }^{*}$ über grundsätzliche Aufgaben der Verteidigung vom 8. 9.1942 und Operationsbefehl Nr. 1, gez. Adolf Hitler, über die weitere Kampfführung im Osten vom 14. 10. 1942, beide Dokumente sind abgedruckt in: KTB OKW, Bd. II/2, S. 1292-1297 und S. 1301-1304; AUSSME, L 13/48-6, Maximilian Freiherr von Weichs (Ia Nr. 4469/42 geh. Kdos.) an die Oberbefehlshaber der 2. ungarischen und der 8. italienischen Armee vom 6. 12. 1942 (Auszug).

265 BA-MA, MFB4 41403, Bl. 1041 f., KTB des Deutschen Generals beim italienischen AOK 8, Eintrag vom 14. 10. 1942; MFB4 18276, Bl. $392 \mathrm{ff}$., Deutscher General beim italienischen AOK 8 (Nr. 835/42 geh.) vom 30.11. 1942: Besondere Führungsanordnungen für DVSt 
die den Vorstellungen der Verbündeten entsprochen hätte. Die taktisch gesehen durchaus richtigen deutschen Empfehlungen fanden so ihre Grenzen nicht nur in der Beratungsresistenz der Verbündeten, sondern auch in den strukturellen Schwächen des königlichen Heeres, von Sachzwängen, die die breiten Verteidigungsabschnitte mit sich brachten, gar nicht zu reden. So blieb es weitgehend bei einem Stützpunktsystem italienischer Prägung, wobei man freilich nur die vorderen Stellungen einigermaßen hatte ausbauen können ${ }^{266}$. Projekte zur Anlage einer zweiten Linie oder zur Befestigung wichtiger Verkehrsknotenpunkte und Nachschubbasen wie Djogtewo, Kantemirowka oder Rossosch kamen über bescheidene Anfänge nur selten hinaus.

\section{2. „Kleiner Saturn “ und „Ostrogoschsk-Rossosch“. Die Offensiven der Roten Armee und die Zerschlagung der ARMIR}

Als die Rote Armee im Winter 1942 zum großen Gegenschlag ausholte, blieben die italienischen Divisionen zunächst unbehelligt. Die am 19. November entfesselte Offensive mit dem Decknamen "Uranus" zielte auf die nördlich und südlich von Stalingrad dislozierten Verbände der 3. und 4. rumänischen Armee, deren Stellungen rasch durchbrochen werden konnten. Bereits am 23. November vereinigten sich die Spitzen der beiden sowjetischen Stoßkeile im Rücken der 6. Armee, die damit zusammen mit Teilen der 4. Panzerarmee eingeschlossen war ${ }^{267}$. Die Rückwirkungen dieser Ereignisse auf die 8. italienische Armee waren gravierend. Durch den Zusammenbruch der 3. rumänischen Armee hing nämlich nicht nur der rechte Flügel des XXIX. Armeekorps in der Luft, sondern die ARMIR verlor auch nahezu alle deutschen Großverbände, die in ihrem Abschnitt als "Korsettstangen “ an der Front eingesetzt oder als Reserven vorgesehen waren. Die 22. Panzerdivision war schon eine Woche vor Beginn des Unternehmens "Uranus“ dem XXXXVIII. Panzerkorps unterstellt worden und operierte seither im Abschnitt der Rumänen, während die 62. und die 294. Infanteriedivision nach den ersten sowjetischen Erfolgen eilig nach Süden marschierten, um im Rahmen der neugebildeten Gruppe Hollidt die bedrohliche Lage bei der 3. rumänischen Armee zu stabilisieren. Da die 3. schnelle Division die Stellungen der 62. Infanteriedivision übernehmen mußte, verfügte das italienische Armeeoberkommando praktisch über keine Reserven mehr ${ }^{268}$. Einzig die im Verband des XXXV. italienischen Armeekorps eingesetzte 298. Infanteriedivision verblieb im Befehlsbereich von Generaloberst Gariboldi, doch dieser Verband hatte mit seinen durch ein sogenanntes Turk-Bataillon verstärkten beiden Infanterieregimentern nicht viel mehr zu bieten als eine der italieni-

und DVK Nr. 4. Ein italienischer Stützpunkt wird beschrieben bei Rigoni Stern, Alpini im russischen Schnee, S. 7-23.

266 AUSSME, DS II 973, KTB II. Armeekorps, September/Oktober 1942, Anlage 281: Karte zum Stand der Arbeiten an den Verteidigungsstellungen zum 1. 10. 1942; L 14/87-1, Comando $8^{2}$ Armata, Ufficio Operazioni: La $2^{2}$ battaglia difensiva del Don, Teil 1: La preparazione dell'8a Armata, S. 21.

267 Vgl. Wegner, Krieg gegen die Sowjetunion, in: DRZW 6, S. 997-1023.

268 Vgl. Kehrig, Stalingrad, S. 105 und S. 235; Operazioni delle unità italiane al fronte russo, S. 318 . 
schen Divisionen, wenn man von ihrer besseren Ausstattung mit schweren Panzerabwehrkanonen einmal absieht ${ }^{269}$.

Während die neugebildete Heeresgruppe Don Ende November/Anfang Dezember 1942 verzweifelt versuchte, den Vormarsch der Roten Armee aufzuhalten und die Voraussetzungen für einen Vorstoß nach Stalingrad zu schaffen, verharrten die Divisionen der nach wie vor der Heeresgruppe B unterstehenden ARMIR in ihren Stellungen. Die sowjetische Führung plante dagegen bereits ihre nächste große Offensive im Süden der Ostfront. Das Unternehmen "Saturn “ sah einen Angriff am mittleren Don vor und zielte nach der Vernichtung der deutsch-rumänischen Armeeabteilung Hollidt und der 8. italienischen Armee auf Rostow, um neben dem Rest der 4. Panzerarmee auch die im Kaukasus stehende Heeresgruppe A von ihren rückwärtigen Basen abzuschneiden. Logistische und operative Probleme erzwangen jedoch eine Änderung der Pläne, wobei sich das nun unter dem Decknamen „Kleiner Saturn“ laufende Unternehmen unter Verzicht auf einen Vorstoß in die Tiefe des Raumes vor allem gegen die Heeresgruppe Don richtete und alle Versuche unmöglich machen sollte, die an der Wolga eingeschlossenen Verbände zu entsetzen ${ }^{270}$.

Auf deutscher Seite wurden seit Ende November Angriffe gegen die 8. Armee befürchtet, deren Frontabschnitt man nicht nur für exponiert, sondern aufgrund der unterstellten Unzuverlässigkeit der italienischen Divisionen auch für besonders anfällig hielt ${ }^{271}$. Doch genaueres war weder dem OKW noch dem Deutschen General bekannt, der die anhaltenden feindlichen Truppenbewegungen am 2. Dezember zwar als Vorbereitungen für einen Angriff deutete, dessen Zielsetzung jedoch nicht darin sah, in die „Tiefe durchzustoßen“, sondern lediglich darin, die „eigene Gliederung festzustellen und Brückenköpfe zu bilden “272. Noch unmittelbar vor Beginn des Großangriffs meldete General von Tippelskirch an den Generalstabschef des Heeres, es zeichne sich keine Durchbruchsabsicht ab, vielmehr bestehe der Eindruck, es gehe vor allem darum, die eigenen Kräfte zu binden ${ }^{273}$. Allerdings hielt cs die deutsche Führung auch ohne konkrete Kenntnisse der sowjetischen Absichten für angebracht, den von allen deutschen Reserven entblößten Sektor der 8. Armee wieder zu verstärken. So bemühte sich die Heeresgruppe B seit dem 26. November darum, der ARMIR deutsche Panzerjäger zuzuführen ${ }^{274}$, und im OKW wurde am 30. November nach einem Wutanfall Hitlers über die mangelhafte Ausrüstung der Verbündeten beschlossen, sowohl die Front der 2. ungarischen Armee als auch die

269 BA-MA, MFB4 18275, Bl. 1205 f., Zustandsbericht 298. Infanteriedivision vom 5. 12. 1942. An Panzerabwehrwaffen verfügte die Division über 35 leichte, 16 mittlere und 15 schwere Pak; MFB4 18276, Bl. 279, Fernschreiben des AOK 8 (Nr. 02/6530 - gez. Bruno Malaguti) an die Heeresgruppe B vom 30.11. 1942.

$270 \mathrm{Vgl}$. Wegner, Krieg gegen die Sowjetunion, in: DRZW 6, S. $1050 \mathrm{f}$.

271 Vgl. KTB OKW, Bd. II/2, S. 1054, Eintrag vom 30.11.1942, und Lagebesprechungen, S. 48 f. (Mittagslage vom 12. 12. 1942); vgl. auch Förster, Ruolo, in: Italiani sul fronte russo, S. $245 \mathrm{ff}$.

272 BA-MA, MFB4 41403, Bl. 1055, KTB des Deutschen Generals beim italienischen AOK 8, Eintrag vom 2. 12. 1942.

273 BA-MA, MFB4 41403, Bl. 1068, KTB des Deutschen Generals beim italienischen AOK 8, Eintrag vom 15.12.1942; vgl. auch KTB OKW, Bd. II/2,S.1128, Eintrag vom 13.12.1942. Erst ein am 15. 12. 1942 aufgefundener Operationsbefehl deckte die sowjetischen Absichten auf.

274 BA-MA, MFB4 41403, Bl. $1053 \mathrm{ff}$. und Bl. 1058, KTB des Deutschen Generals beim italienischen AOK 8, Einträge vom 26. 11., 27. 11., 30. 11., 1. 12., 7. 12. und 9. 12. 1942. 
der 8. italienischen Armee durch deutsche Verbände zu stützen ${ }^{275}$. Bis zum 10. Dezember waren unter anderem die 385. Infanteriedivision mit zwei Regimentern, die schwache 27. Panzerdivision, das Grenadierregiment 318 der 213. Sicherungsdivision, das Polizeiregiment 14 und mehrere Abteilungen beziehungsweise Kompanien Panzerjäger in den Sektor der ARMIR - und hier vor allem zum II. Armeekorps - verlegt worden; weitere Verbände wie die SS-Brigade Schuldt und das Führerbegleitbataillon befanden sich auf dem Marsch ${ }^{276}$.

Am 11. Dezember begann die Rote Armee damit, ihre Offensive vorzubereiten, indem sie immer wieder Angriffe in Bataillonsstärke gegen das Zentrum der italienischen Aufstellung vortrug. Die Schwerpunkte lagen dabei zwischen Nowaja Kalitwa und Samodurowka (Division "Cosseria“), bei Deresowka (Grenadierregiment 318), im Raum Werch. Mamon (Division "Ravenna ") und im Don-Bogen von Ogolew (Division "Pasubio“) ${ }^{277}$. Noch ging es nicht darum, einen operativen Durchbruch zu erzielen, sondern darum, die in den angegriffenen Abschnitten eingesetzten italienischen und deutschen Einheiten soweit wie möglich zu schwächen. Diese Abnutzungsschlacht dauerte fünf Tage und tobte insbesondere im Abschnitt des II. Armeekorps, wo neben den Divisionen "Cosseria“ und „Ravenna“ auch das deutsche Grenadierregiment 318 stand.

Wenn die Angreifer einen Einbruch erzielen konnten, wurden sie durch Gegenstöße wieder vertrieben. Dabei warfen die Führungsstäbe nach und nach alle taktischen Reserven ins Gefecht, wobei diese ebenso wie die zur Unterstützung eingesetzten deutschen Kampfgruppen ${ }^{278}$ schwere Verluste erlitten. „Italiener werden aus Stützpunkten herausgeschossen und machen teure Gegenangriffe“, vermerkte das Kriegstagebuch des Deutschen Generals. „Mangelhafter Stellungsausbau“ und "mangelhaftes Zusammenwirken mit schweren Waffen" und Artillerie führten jeden Tag erneut zu "Geländeverlusten“. Die dann folgenden "Versuche, mit schneidigen Gegenstößen Lage wieder herzustellen", forderten einen hohen Blutzol1279. Doch cum grano salis stellten die deutschen Verbindungsoffiziere den italienischen Truppen in dieser Phase der Schlacht ein gutes Zeugnis aus. Am Ende des dritten Kampftages meldete der Verbindungsstab zum II. Armeekorps, die "Ravenna“ habe

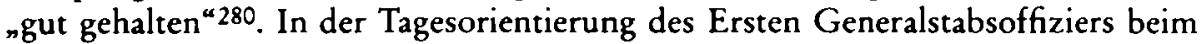

275 Vgl. KTB OKW, Bd. II/2, S. 1054, Eintrag vom 30. 11. 1942.

276 AUSSME, L 13/48-8, Aufstellung des AOK 8 über die bis zum 10. 12. 1942 in den Sektor der Armee verlegten deutschen Verbände; BA-MA, MFB4 18275, Bl. 556, Deutscher General beim italienischen AOK 8: Ia-Tagesmeldung vom 9. 12. 1942; vgl. Lagebesprechungen, S. 49 (Mittagslage vom 12.12.1942).

277 Vgl. Operazioni delle unità italiane al fronte russo, S. 338-354 und Abbildung 38.

278 Die italienischen Stäbe waren in der Verwendung der deutschen Reserven jedoch nicht frei; BA-MA, MFB4 18276, Bl. 331 f., Heeresgruppe B (Ia - Nr. 4502/42 geh. Kdos.) an AOK 8 vom 8. 12.1942.

279 BA-MA, MFB4 41403, Bl. 1068 f., KTB des Deutschen Generals beim italienischen AOK 8, Eintrag vom 15. 12. 1942; General von Tippelskirch äußerte sich gegenüber General Marras (ebenda, Bl. 1107) am 5. 1. 1943 ähnlich: "Die italienische Armee kämpfe zu teuer. Sie habe nahezu alle Reserven in den ersten Tagen der kleinen russischen Angriffe durch meist mit Bravour, aber unter hohen Verlusten geführte Gegenstöße verbraucht." Vgl. auch den Wochenbericht des Italien-Referats der Attaché-Abteilung im OKH an den deutschen Militärattaché in Rom vom 16.1.1943, abgedruckt in: Förster, Stalingrad, S. $143 \mathrm{ff}$.

280 BA-MA, MFB4 18275, Bl. 536, Deutscher General beim italienischen AOK 8: Ia-Tagesorientierung vom 15.12. 1942. 
Deutschen General vom 15. Dezember war zu lesen, das „Verhalten der eigenen Truppe" verdiene "Anerkennung “, und noch in der $Z$ wischenmeldung vom 16 . Dezember hieß es: „Große Teile der Inf[anterie] der Ravenna hielten tapfer die alten Stellungen in einzelnen Stützpunkten. "281 Freilich meldete die 27. Panzerdivision schon am 14. Dezember, zwar zeichneten sich noch keine Durchbrüche ab, wenn aber der „Russe mit grösseren [Panzer-]Kräften“ komme, dann könne "er nicht aufgehalten werden “282. Tatsächlich waren zumindest die Infanteristen der „Cosseria“ fast am Ende ihrer Kräfte, auch wenn ihre Moral noch nicht gebrochen war. Im Kriegstagebuch der Division heißt es:

"Nach vier Tagen des ununterbrochenen und blutigen Kampfes befinden sich die Verbände an der Grenze der menschenmöglichen Widerstandskraft. Dessen ungeachtet behauptet sich bei allen, bei Führern wie bei einfachen Soldaten, der höchste Wille, Widerstand zu leisten und an Ort und Stelle zu sterben. ${ }^{283}$

Bei der Heeresgruppe B reagierte man am Nachmittag des 15. Dezember auf die immer schwierigere Lage im Sektor des II. Korps mit dem Befehl, die Division "Cosseria“ zur Verstärkung der "Ravenna “ vom linken Flügel abzuziehen und dafür die 385. Infanteriedivision einzusetzen, die am folgenden Tag um sechs Uhr den Abschnitt der „Cosseria“ übernehmen sollte ${ }^{284}$.

Diese Bewegung ließ sich jedoch nicht mehr wie vorgesehen vollziehen ${ }^{285}$, da am 16. Dezember bei Tagesanbruch die eigentliche Offensive der Roten Armee begann; die Abnutzungsschlacht wurde zur Durchbruchsschlacht ${ }^{286}$. Wieder lagen die Schwerpunkte bei der Division „Pasubio“ und im Bereich des II. Armeekorps, wo die 6. sowjetische Armee (Woronesch-Front) und die 1. Gardearmee (SüdwestFront) mit nicht weniger als zehn Schützendivisionen, 13 Panzerbrigaden, zwei Panzerregimentern und vier motorisierten Schützenbrigaden zum Angriff angetreten waren. Dabei sah der Operationsplan vor, das Zentrum und den rechten Flügel der 8. Armee zunächst frontnah zu umfassen und dann im Zusammenwirken mit der aus dem Sektor der Armeeabteilung Hollidt angreifenden 3. Gardearmee auch weiträumig einzuschließen. Die Anfangserfolge der Roten Armee waren beachtlich, aber nicht durchschlagend. Dichter Nebel behinderte den Einsatz von Artillerie und Kampfflugzeugen, Panzer wurden von Minenfeldern aufgehalten, und die italienische Infanterie wehrte sich verzweifelt ${ }^{287}$. Allerdings wurden ihre Stützpunkte einer nach dem anderen eingeschlossen, zusammengeschossen oder von Panzern

281 BA-MA, MFB4 18275, Bl. 534, Deutscher General beim italienischen AOK 8: la-Zwischenmeldung vom 16. 12. 1942. Vgl. auch die um Differenzierung bemühten Urteile in den Dok. 8,9 und 10 des vorliegenden Bandes.

282 BA-MA, MFB4 41403, Bl. 1066, KTB des Deutschen Generals beim italienischen AOK 8, Eintrag vom 14. 12. 1942.

${ }^{283}$ AUSSME, DS II 1094, KTB Division „Cosseria“, November/Dezember 1942, Eintrag vom 15. 12.1942.

284 BA-MA, MFB4 41403, Bl. 1070, KTB des Deutschen Generals beim italienischen AOK 8, Eintrag vom 15. 12. 1942.

285 BA-MA, MFB4 41403, Bl. 1072, KTB des Deutschen Generals beim italienischen AOK 8, Eintrag vom 16. 12. 1942.

286 Zum Verlauf der Kämpfe und zum Kräfteverhältnis vgl. Operazioni delle unità italiane al fronte russo, S. 324-335, S. 354-387 und Abbildung 36; die hier angegebenen Zahlen sind sowjetischer Provenienz.

${ }^{287}$ Vgl. Plotnikov, Offensiva delle truppe sovietiche, in: Italiani sul fronte russo, S. 526. 
niedergewalzt ${ }^{288}$, und am frühen Nachmittag meldete der Erste Generalstabsoffizier der 8. Armee, Oberstleutnant Bonzani, die gesamte Infanterie der Division "Ravenna" sei "überrollt" worden289. Gleichwohl gelang es gepanzerten Kampfgruppen, den sowjetischen Vormarsch noch einmal aufzuhalten, doch es fehlte an Infanterie, um die Bresche zu schließen, die sich bei der Division "Ravenna“ aufgetan hatte. Tatsächlich war die "Ravenna" am Ende. Am Nachmittag des 16. Dezember vermerkte das Kriegstagebuch des Deutschen Generals erstmals "fluchtartig“ zurückgehende italienische Soldaten, und der Kommandeur der Division ${ }_{n}$ Ravenna ${ }^{*}$, General Dupont, mußte zugeben, daß er seine Leute "nicht mehr in der Hand" habe ${ }^{290}$.

Doch das eigentliche Drama stand noch bevor. Am 17. Dezember warf die Rote Armee die Masse ihrer im Brückenkopf von Werch. Mamon konzentrierten Panzer in die Schlacht, und diesmal war alle Gegenwehr vergeblich. Weder die Reste der italienischen Regimenter noch die in diesem Raum eingesetzten deutschen Truppen vermochten die Angriffe zum Stehen zu bringen, so daß die rechts und links von der Frontlücke kämpfenden Verbände des II. und des XXXV. Armeekorps von der Umfassung bedroht waren und ihre inneren Flügel immer weiter zurücknehmen mußten. Als sich der Einbruch, den die sowjetischen Einheiten am Vortag in die Stellungen des II. Armeekorps erzielt hatten, zum Durchbruch ausweitete, war das deutsche Kalkül gescheitert, die Front so lange zu halten, bis Reserven herangekommen waren, um die Lage zu bereinigen und so gefährliche Rückwirkungen auf den Vorstoß des LVII. deutschen Panzerkorps zu vermeiden, das am 12. Dezember zum Entsatz von Stalingrad angetreten war291. Zwar hatte man Truppen in Marsch gesetzt, aber sowohl das Generalkommando des XXIV. Panzerkorps als auch die 387. Infanteriedivision kamen zu spät, um noch wirksam in die Durchbruchsschlacht eingreifen zu können. Obwohl auch an diesem Tage noch Widerstand geleistet wurde, begann sich im Laufe des 17. Dezember bei den italienischen Soldaten stellenweise Panik breit zu machen ${ }^{292}$, die in einen ebenso unautorisierten wie ungeordneten Rückzug mündete und auch deutsche Einheiten in Mitleidenschaft zog. Der Zusammenhalt der Verbände ging ebenso verloren wie die Verbindung zwischen den Stäben und der fechtenden Truppe, so daß die Führung kein klares Bild der Lage mehr gewinnen konnte und die Offiziere an der Front ohne eindeutige $\mathrm{Be}$ fehle blieben.

Wie schon Ende August bei der Division „Sforzesca“ so zeigte sich auch jetzt wieder, daß die italienischen Infanteristen verbissen aushielten, solange sie in ihren Stellungen lagen, daß sie aber rasch die Orientierung verloren, wenn diese aufgegeben werden mußten. $\mathrm{Zu}$ viele mangelhaft ausgebildete junge Truppenoffiziere wa-

$288 \mathrm{Vgl.} 8^{\text {a }}$ Armata Italiana nella seconda battaglia difensiva del Don, S. 22.

289 BA-MA, MFB4 41403, Bl. 1074, K'TB des Deutschen Generals beim italienischen AOK 8, Eintrag vom 16. 12. 1942; zur Lücke zwischen Deresowka und Gadjutsche vgl. ebenda, Bl. 1073-1076.

290 BA-MA, MFB4 41403, Bl. 1075f., KTB des Deutschen Generals beim italienischen AOK 8, Eintrag vom 16. 12.1942.

291 Vgl. Förster, Ruolo, in: Italiani sul fronte russo, S. 248, und Kehrig, Stalingrad, S. 354-369.

292 BA-MA, MFB4 41403, Bl. 1082 und Bl. 1084, KTB des Deutschen Generals beim italienischen AOK 8, Einträge vom 17. 12. und 18.12. 1942; zum folgenden vgl. auch ebenda, Bl. 1084-1091, Einträge vom 18.-20. 12. 1942. 
ren nicht in der Lage, aus eigener Initiative die richtigen Entscheidungen zu treffen. Sie hätten es zwar verstanden zu sterben, seien aber unfähig gewesen, Befehle zu geben, wie Generaloberst Gariboldi nach der Schlacht bemerkte ${ }^{293}$. Diese Offiziere hätten von oben gut geführt und aus dem Unteroffizierskorps unterstützt werden müssen ${ }^{294}$. Doch da in den Bataillons- und Regimentsgefechtsständen nicht selten Reserveoffiziere den Ton angaben, deren Fähigkeiten sehr zu wünschen übrig ließen, und erfahrene Unteroffiziere im königlichen Heer ein knappes Gut waren, blieben die Zugführer und Kompaniechefs in ihren Stützpunkten am Don nur allzuoft auf sich allein gestellt. Der Truppe entging die mangelnde Professionalität ihrer Führer nicht, was nicht gerade dazu beitrug, ihre Autorität und ihr Ansehen zu fördern. Ein Unteroffizier der Bersaglieri vertraute beispielsweise seinem Tagebuch an, sein Vorgesetzter habe mit einem Offizier lediglich die Uniform gemeinsam ${ }^{295}$, und ein Kamerad bemerkte bissig, die eigenen Offiziere seien ebenso desorientiert wie uninformiert, während man genau wisse, was zu tun sei, wenn die Deutschen den Befehl führten ${ }^{296}$. Dies waren alles andere als gute Voraussetzungen für kritische Situationen, und tatsächlich gingen mutige, ja todesverachtende Akte individueller Tapferkeit im beginnenden Chaos unter, als der Großangriff der Roten Armee erste durchschlagende Erfolge zeigte. Zu diesen strukturellen Mängeln gesellte sich zumindest bei den drei Divisionen, die mit dem CSIR an die Ostfront gekommen waren, ein weiteres Problem: der Austausch der Offiziere und Soldaten, die schon länger als ein Jahr auf diesem Kriegsschauplatz eingesetzt waren. Damit kamen zwar frische Kräfte an die Front, doch der Verlust an Erfahrung und innerem $\mathrm{Zu}$ sammenhalt war unübersehbar ${ }^{297}$. Aus kämpfenden Soldaten wurden unter diesen Umständen schnell Versprengte, die man hinter der Front nur mit Mühe sammeln und wieder ins Gefecht schicken konnte, zumal viele keine Waffe mehr trugen.

Das Oberkommando der Heeresgruppe B reagierte auf die sich zuspitzende Lage neben detaillierten Befehlen zunächst mit Führungshilfen, die in der Entsendung von Oberst Eberhard Kinzel zum II. Armeekorps gipfelten ${ }^{298}$. Dieser erfahrene Generalstabsoffizier übernahm am 15. Dezember nicht nur den Befehl über das Verbindungskommando, sondern sollte auch und vor allem die Entscheidungen von General Zanghieri und seines Generalkommandos im Sinne der deutschen Führung beeinflussen. Dann versuchten sowohl Generaloberst von Weichs als auch General von Tippelskirch, bis zum 17. Dezember eher durch Lob als durch Tadel, Durchhaltewillen, Kampfmoral und Zuversicht des Armeeoberkommandos zu stärken ${ }^{299}$. Doch als alle Versuche, Ordnung in die Reste des II. Armeekorps zu bringen und neue Haltelinien zu beziehen, ohne befriedigendes Ergebnis blieben, wurden Ton und Methoden rauher. Am Nachmittag des 17. Dezember erzwang die Heeres-

293 Vgl. Dok. 18, S. 188.

29+ AUSSME, DS II 1551/1, Aufzeichnungen von Generaloberst Italo Gariboldi über die 8. Armee im Krieg gegen die Sowjetunion vom Dezember 1946, S. 39-42.

295 AUSSME, L 13/161, Tagebuch von Francesco Zito, Eintrag vom 21. 3. 1942.

296 ACS, MIn, DGPS - Divisione Polizia Politica, busta 215, fasc. 2: Corpo di Spedizione Militare, Bericht aus Florenz vom 8. 10. 1942.

297 BA-MA, MFB4 18035, Bl. 263 ff., Notiz von Major Fellmer über Gespräche mit dem Generalstabschef und dem Ersten Generalstabsoffizier der 8. Armee am 21. 12. 1942.

298 BA-MA, MFB4 41403, Bl. 1216, KTB DVK II. Armeekorps, Eintrag vom 14. 12. 1942.

299 BA-MA, MFB4 41403, Bl. 1081 f., KTB des Deutschen Generals beim italienischen AOK 8 , Eintrag vom 17. 12. 1942. 
gruppe B weitreichende Vollmachten für Oberst Kinzel zur Organisation des weiteren Widerstandes im Sektor des II. Armeekorps ${ }^{300}$. Einen Tag später drängte Oberst Winter, der Erste Generalstabsoffizier der Heeresgruppe, General von Tippelskirch,

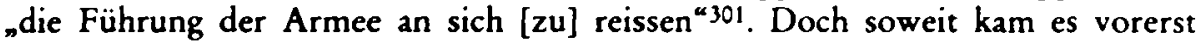
nicht ${ }^{302}$, auch wenn wichtige Befehle am Armeeoberkommando vorbei erteilt wurden und das Generalkommando des XXIV. Panzerkorps am 18. Dezember die Verantwortung für den Abschnitt des II. Armeekorps und alle dort kämpfenden Truppenteile übernahm ${ }^{303}$. Auch verbal setzte die deutsche Führung nun auf Pression. Generaloberst Gariboldi mußte sich etwa die Drohung anhören, wenn keine energischen Schritte unternommen würden, den Auflösungserscheinungen Herr zu werden, werde man dem „Führer" melden, „daß die ital. Armee nicht mehr kämpfe, sondern wegginge " ${ }^{304}$. Und der Erste Generalstabsoffizier der ARMIR sah sich mit dem zornigen Ausruf des Deutschen Generals konfrontiert, es sei unmöglich, daß fünf italienische Divisionen „bis zum Schwarzen Meer ausreissen “ 305.

Letztlich waren all diese Interventionen ein Ausdruck des hilflosen Bemühens, das Unmögliche doch noch möglich zu machen. Aber abgesehen davon, daß derartige verbale Ausfälle das immer wackligere Fundament der deutsch-italienischen Waffenbrüderschaft in gefährlicher Weise unterminierten, bewirkten sie nichts, da die Rote Armee den Verteidigern immer mindestens einen Schritt voraus war und mit raschen Vorstößen alle Versuche zunichte machte, die Lage zu stabilisieren ${ }^{306}$. Am 17. Dezember bedrohten die sowjetischen Panzerspitzen mit dem Flußtal des Bogutschar ein wichtiges Einfallstor in den Rücken der italienischen Front. Zudem hatte sich im Abschnitt der 3. schnellen Division (XXIX. Armeekorps) auf dem rechten Armeeflügel eine gefährliche Situation ergeben, die bei einem Durchbruch eine beidseitige Umfassung dreier Korps befürchten ließ. Am 18. Dezember standen die Angreifer vor Taly, wo sich noch bis vor kurzem das Hauptquartier des II. Armeekorps befunden hatte, am 19. Dezember erreichten sie Kantemirowka, eines der vorgeschobenen logistischen Zentren der Intendenza, und Tschertkowo. Damit stand die Rote Armee aber nicht nur tief im Rücken des XXXV. und des

300 BA-MA, MFB4 41403, Bl. 1083, KTB des Deutschen Generals beim italienischen AOK 8, Eintrag vom 17. 12. 1942; vgl. auch Heeresgruppe B (Ia Nr. 4615/42 geh. - gez. Maximilian Freiherr von Weichs) an das AOK 8 vom 17. 12.1942, abgedruckt in: Operazioni delle unità italiane al fronte russo, S. $696 \mathrm{f}$.

301 BA-MA, MFB4 41403, Bl. 1084, KTBB des Deutschen Generals beim italienischen AOK 8, Eintrag vom 18. 12. 1942. Zwar wird von Tippelskirch mit den Worten zitiert "darüber kein

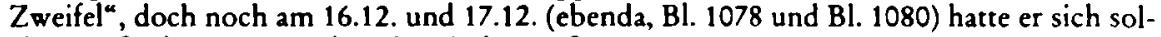
chen Maßnahmen gegenüber skeptisch geäußert.

302 Die deutschen Einheiten wurden von Tippelskirch erst am Morgen des 30.12. 1942 unterstellt; BA-MA, MFB4 41403, Bl. 1101, KTB des Deutschen Generals beim italienischen AOK 8, Eintrag vom 30. 12. 1942.

303 Vgl. 82 Armata Italiana nella seconda battaglia difensiva del Don, S. 32; BA-MA, MFB4 41403, Bl. 1085 f., KTB des Deutschen Generals beim italienischen AOK 8, Eintrag vom 18. 12. 1942.

304 BA-MA, MFB4 18035, Bl. 269, Notiz des Deutschen Generals beim italienischen AOK 8 vom 19. 12.1942.

305 BA-MA, MFB4 41403, Bl. 1090, KTB des Deutschen Generals beim italienischen AOK 8, Eintrag vom 20.12. 1942.

306 Hierzu und zum folgenden vgl. $8^{2}$ Armata Italiana nella seconda battaglia difensiva del Don, S. 22-32. 
XXIX. Armeekorps, sondern hatte auch die wichtige Eisenbahnverbindung zwischen Millerowo und Rossosch unterbrochen. Als sich zwei Tage später die nach Südwesten marschierenden sowjetischen Verbände bei Djogtewo mit den Panzerspitzen der aus dem Sektor der Armeeabteilung Hollidt vorstoßenden 3. Gardearmee trafen, waren vier italienische Divisionen und eine deutsche eingeschlossen.

Zwar war schon am 19. Dezember der Befehl gegeben worden, die Stellungen zurückzuverlegen, doch weder an der Tichaja noch am Tschir ließ sich - wie beabsichtigt - eine neue Verteidigungslinie aufbauen. Schließlich ging es nur noch darum, sich zu den eigenen Linien durchzuschlagen, wobei die Verbände des XXXV. und des XXIX. Armeekorps (oder was davon noch übrig war) zwei Marschkolonnen bildeten. Der blocco nord bestand vor allem aus Teilen der Divisionen "Ravenna“, "Pasubio" und „Torino" sowie der 298. deutschen Infanteriedivision, der blocco sud aus Teilen der Divisionen „Pasubio“, „Sforzesca“ und Celere sowie der SS-Brigade Schuldt. Die nördliche Kolonne schlug sich bis Weihnachten zunächst nach Tschertkowo durch und wurde Mitte Januar bei Belowodsk von eigenen Einheiten aufgenommen, während die südliche Kolonne nach einer wahren Odyssee in den letzten Dezembertagen bei Skasyrskaja auf deutsche Truppen stieß und von dort in mehreren Tagesmärschen nach Forschtadt am Donez weiterzog ${ }^{307}$.

Währenddessen hielt das durch die Division „Vicenza“ verstärkte Alpinikorps weiterhin seine alten Stellungen am Don, wobei es die Alpini der Division "Julia“ fertiggebracht hatten, zusammen mit den zusammengewürfelten Truppen des XXIV. Panzerkorps südwestlich des Flusses Kalitwa eine improvisierte Verteidigungslinie aufzubauen. Doch Mitte Januar 1943 brach das Verhängnis auch über die Alpini herein. Nach den Unternehmen "Uranus“ und „Kleiner Saturn“ startete die Rote Armee am 12. Januar mit der Operation „Ostrogoschsk - Rossosch“ eine weitere Großoffensive gegen die Streitkräfte der Achsenmächte im Süden der Ostfront. Dabei sollten dic 40. Armec (Woronesch-Front), die 3. Panzerarmee und die 6. Armee (Südwest-Front) die am Don verbliebenen deutschen, ungarischen und italienischen Verbände zerschlagen, die Bahnlinie Swoboda - Kantemirowka unter Kontrolle bringen und darüber hinaus bis auf die Linie Urazowo - Alexejewka - Repjewka nach Westen vorstoßen ${ }^{308}$.

Zunächst richtete sich der Angriff gegen die 2. ungarische Armee, deren Stellungen am 14. Januar durchbrochen wurden. Am Morgen desselben Tages begann die Offensive gegen das XXIV. Panzerkorps, und auch hier hielten die Linien nicht lange. Allerdings erkannten weder die deutschen noch die italienischen Führungsstäbe die Tragweite dieses Durchbruchs, so daß gepanzerten Truppenteilen nicht nur ein Handstreich gegen das Generalkommando des XXIV. Panzerkorps, sondern am 15. Januar auch ein überraschender Vorstoß auf Rossosch gelang, wo sich

307 Vgl. Operazioni delle unità italiane al fronte russo, S. 387-400 und S. 406-414; die Teile der Division „Ravenna“, die hatten herausgezogen und neu geordnet werden können, wurden vom 22.12. 1942-24. 1. 1943 bei Woroschilowgrad am Donez eingesetzt, die "Cosseria“" sammelte sich dagegen zunächst im Abschnitt des Alpinikorps und trat dann den Marsch nach Westen an; vgl. ebenda, S. 415-421.

308 Vgl. hierzu und zum folgenden Massignani, Alpini e tedeschi, S. 53-89 und S. 96ff.; Schreiber, Italiens Teilnahme, in: Förster (Hrsg.), Stalingrad, S. 276 ff.; wertvolle Details zum Verlauf der sowjetischen Offensive finden sich bei Wimpffen, Zweite ungarische Armee, passim. 
das Hauptquartier und die Versorgungsbasis des Alpinikorps befanden. Auch wenn dieser Vorstoß zurückgeschlagen werden konnte, so war doch klar, daß das XXIV. Panzerkorps in zwei Teile gespalten war und sowjetische Verbände tief in der Flanke beziehungsweise im Rücken der Alpini standen. Der Rückzugsbefehl ließ dennoch bis zum 17. Januar auf sich warten, und als sich die Divisionen „Tridentina", "Julia", "Cuneense" und "Vicenza" zusammen mit der 385. und 387. deutschen Infanteriedivision auf den Weg nach Westen machten, waren sie bereits eingeschlossen. Alpini und Grenadiere mußten sich ihren Weg freikämpfen, wobei die einzelnen Kolonnen ein höchst unterschiedliches Geschick erwartete. Während die zusammen mit den wenigen verbliebenen schweren Waffen des XXIV. Panzerkorps an der Spitze marschierende "Tridentina" den sowjetischen Sperriegel schließlich am 26. Januar bei Nikolajewka durchbrechen konnte und einige Tage später auf sicheres Terrain gelangte, erreichten nur Reste der anderen Divisionen die eigenen $\mathrm{Li}$ nien. Das Gros war gefangengenommen worden oder tot.

\section{Der Rückzug als militärisches und bündnispolitisches Desaster}

Der Rückzug vom Don mit all seinen Schrecken wurde nicht nur zu einem Schlüsselerlebnis für die überlebenden Soldaten der ARMIR, sondern er sollte nach dem Krieg das Bild fast vollständig beherrschen, das man sich in Italien von der Campagna di Russia machte. Auf dem fluchtartigen Rückzug nach Westen spielten sich unbeschreibliche Szenen ab, wobei deutsche und italienische Soldaten nicht selten mit der Waffe in der Hand um Fahrzeuge, Lebensmittel oder Unterkünfte stritten. Nach dem weitgehenden Zusammenbruch der militärischen Ordnung regierte das Gesetz des Stärkeren mit brutaler Härte. Doch für das Verhältnis der Verbündeten erwies es sich als fast noch gravierender, daß sich die zuständigen deutschen Stellen in der Behandlung der überlebenden italienischen Soldaten, die sich als Helden fühlten, aber wie Versager oder Feiglinge behandelt wurden, nicht gerade durch große Fürsorge auszeichneten, als das Schlimmste überstanden war. Während der Schlacht am Don und sogar noch zu Beginn des Rückzugs hatte es dagegen immer wieder Szenen gegeben, in denen sich zeigte, daß die deutsch-italienische Waffenbrüderschaft nicht nur auf dem Papier stand, und es dürfte kein Zufall sein, daß das ausführliche Sündenregister, das der Kommandeur des 90 . Infanterieregiments der Division "Cosseria“ den Deutschen im März 1943 zur Last legte, in der Rubrik "fehlende Kooperation im Gefecht" nur einen Vorfall verzeichnet ${ }^{309}$. Dagegen berichtete Brigadegeneral Manlio Capizzi, der Infanterieführer der Division „Ravenna", wie die Kampfgruppe von Major Hoffmann nach dem sowjetischen Durchbruch vom 17. Dezember 1942 "mit Geschick und bewundernswertem, selten anzutreffenden Sinn für Zusammenarbeit " mit ihren Panzern den Rückzug seiner Männer vom Don gedeckt und ihre Stellungen erst dann aufgegeben habe, als auch die letzte italienische Einheit in Sicherheit gewesen sei ${ }^{310}$. Und noch am 19. Dezember waren es deutsche Panzerfahrzeuge, die ein erhebliches Risiko eingingen, als sie Oberstleutnant Luigi De Micheli, den Stabschef der Intendenza, und einige Mitstreiter aus Kantemirowka retteten, das die Italiener am selben Morgen nach dem

309 Vgl. Dok. 24, S. 224.

310 Capizzi, Divisione „Ravenna“, S. 381. 
Erscheinen feindlicher Panzerspitzen überstürzt und in voller Panik aufgegeben hatten ${ }^{311}$.

Ereignisse wie die große Flucht von Kantemirowka, als mehrere Tausend vom Panzerschreck erfaßte italienische Soldaten den Kopf verloren hatten, waren Gift für das deutsch-italienische Verhältnis, da sie alle Vorurteile bestätigten, die man in den Reihen der Wehrmacht gegen das königliche Heer hegte. Während es nur wenige deutsche Augenzeugen gab, die von der Tapferkeit italienischer Infanteristen in ihren Stützpunkten am Don hätten berichten können, gab es zu viele, die in Kantemirowka dabeigewesen waren oder anderswo ähnliche Szenen beobachtet hatten. Dies führte dazu, daß die Italiener nicht mehr als Kameraden und Verbündete, sondern gleichsam als Muster ohne Wert angesehen wurden. Aufmerksamen Beobachtern wie Brigadegeneral Cesare Rossi, dem Infanterieführer der Division „Torino“, entging diese Veränderung nicht:

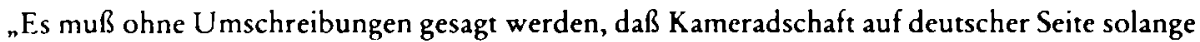
existiert, wie sie kampfbereite, gut bewaffnete Männer zum Gegenstand hat, die in der Lage sind, einen sichtbaren und effektiven Beitrag zu den militärischen Operationen zu leisten. Andernfalls kommt es soweit, daß jedes Gefühl der Brüderlichkeit, der Solidarität und sogar des Respekts vollständig verloren geht. ${ }^{312}$

Aber es waren nicht nur deutsche Soldaten, die ein vernichtendes Urteil über die geschlagenen Verbände der ARMIR fällten. Italienische Offiziere wie Domenico Lo Faso, der in der Führungsabteilung der Division „Sforzesca“ Dienst tat, standen ihnen nur wenig nach. In den Aufzeichnungen des Majors über seine Gedanken beim Vorbeimarsch einer abgekämpften Kolonne ist zu lesen:

${ }_{n}$ Es krampft sich mein Herz zusammen, wenn ich sie vorbeiziehen sehe. Was können wir von Soldaten wie diesen erhoffen? Beim ersten Schuß werden alle abhauen oder sich ergeben. Auch wenn es unter ihnen gute Leute gibt, werden sie doch von der Masse mitgezogen. Mir kommt fast das Heulen vor Wut und Scham. Das ist der italienische Soldat? Wie weit ist es mit unserem Volk gekommen? Und man schämt sich am meisten, wenn man auch Leute zum Vergleich heranzieht, die wir immer als unter uns stehend eingeschätzt haben. Inmitten der unseren ziehen einige Rumänen vorbei. Alle haben ordentliche Uniformen und alle sind bewaffnet, auch diejenigen, die sich mit in Decken gewickelten erfrorenen Füßen langsam hinter den anderen herschleppen. Die Schuld liegt nur bei uns. Wir haben den Subalternoffizieren keine Seele gegeben und wir sind nicht energisch genug gewesen, um sofort gegen die Unordnung und die Unsitte vorzugehen, die Waffen zurückzulassen. Jetzt kann man nur noch leiden. [...] Also ertragen wir diese Schande, um uns daran zu erinnern, wenn es darum gehen wird, die neuen Italiener z.u schaffen. ${ }^{313}$

Der beklagenswerte Zustand der Truppenteile die sich hatten retten können, gab der deutschen Seite freilich noch lange nicht das Recht, ihnen die gebotene Unterstützung zu versagen; im Sinne der gemeinsamen Kriegführung wäre im Rahmen des Möglichen das Gegenteil sinnvoll und ratsam gewesen. Im OKW war man sich dieser Problematik durchaus bewußt und gab gegenüber dem Auswärtigen Amt zu,

311 AUSSME, DS II 1557/1, Bericht von Oberstleutnant Luigi De Micheli über die Ereignisse in Kantemirowka vom 17.-19.12. 1942 für General Biglino.

312 AUSSME, DS II 1555/10, Bericht des Infanterieführers der Division „Torino“, Brigadegeneral Cesare Rossi, über die Ereignisse an der Ostfront zwischen Oktober 1942 und Januar 1943 vom Juni 1943, S. 18 f.

313 Lo Faso, 5 Mesi, S. 101. 
$\mathrm{daß}$ die italienischen „Beschwerden und Wünsche bis zu einem gewissen Grade begründet" seien. Tatsächlich seien die verbündeten Truppen "nach den bekannten Vorkommnissen an der Ostfront an einzelnen Stellen ganz sich selbst überlassen worden. Wenn sie auf ihrem Rückzug ohne Waffen, Verpflegung und alle Hilfsmit-

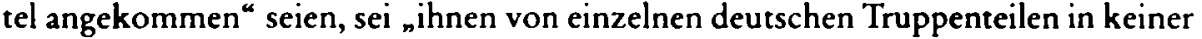
Weise geholfen worden “314. Der Generalstabschef des Heeres hatte General Marras schon am 14. Januar 1943 versichert, er werde "Anweisung geben, daß sich die deutschen Truppen den Truppenteilen der ital. 8. Armee gegenüber besonders kameradschaftlich erweisen", und dem Militärattaché auch in anderen Fragen Entgegenkommen zugesagt ${ }^{315}$; an der Situation vor Ort änderte dies jedoch nicht viel.

Allerdings war es unter den Bedingungen, die zwischen Dezember 1942 und März 1943 im Süden der Ostfront herrschten, zuweilen auch ausgesprochen schwierig, die Bedürfnisse der italienischen Kontingente zu befriedigen, denn die Transport- und Versorgungsdienste hatten genug damit zu tun, die deutschen Gegenangriffe logistisch zu unterstützen. Zudem war ein grundlegender Dissens zwischen dem italienischen Armeeoberkommando und der deutschen Führung unverkennbar. Während letztere nämlich vorwiegend die Nutzung aller verbliebenen Ressourcen zur Stabilisierung der Lage im Auge hatte, bemühten sich die zuständigen italienischen Stäbe vor allem darum, zu retten, was zu retten war - und zwar an Menschen, Material und Kraftfahrzeugen, die die deutschen Kommandos nur zu gerne wieder eingesetzt hätten. Mißverständnisse und Konflikte konnten dabei gar nicht ausbleiben, und als sich Übergriffe von deutscher Seite häuften, ordnete Gariboldi bei Androhung schwerer Strafen an, das Eigentum des königlichen Heeres "mit allen Mitteln zu verteidigen“ 316 .

Letztlich versuchten beide Seiten immer wieder, einander die Schuld für das Desaster in die Schuhe zu schieben. In dieser gespannten Atmosphäre kam es bei den Angehörigen der ARMIR vielfach zu einem überraschend schnellen Wechsel des Feindbilds. Aus den ehemaligen Waffenbrüdern wurden Verräter, die man noch mehr hassen müsse, als man dies im Ersten Weltkrieg getan habe ${ }^{317}$, und aus den zuvor nicht selten mit allen denkbaren Schimpfwörtern bedachten und als rückständig geschmähten Russen und Ukrainern wurden die neinzigen Freunde des italienischen Soldaten " an der Ostfront ${ }^{318}$, wobei man freilich geflissentlich darüber hinwegsah, daß es sich bei der Gastfreundschaft in den Behausungen der Zivilbevölkerung in vielen Fällen um eine "erzwungene Gastfreundschaft " gehandelt hatte ${ }^{319}$. Es gab zwar nach wie vor Truppenteile wie die Kampfgruppe von Oberst Carloni, die auch nach der Katastrophe dazu bereit waren, an der Seite deutscher Soldaten zu kämpfen, und Offiziere, die sich um eine Wiederbelebung der Waffenbrüderschaft

314 ADAP 1918-1945, Serie E, Bd. 5, S. 435 f.: Aufzeichnung des Botschafters z.b.V. Ritter vom 20. 3. 1943.

315 Wochenbericht des Italien-Referats der Attaché-Abteilung im $\mathrm{OKH}$ an den deutschen Militärattaché in Rom vom 16. 1. 1943, abgedruckt in: Förster, Stalingrad, S. 143 ff., hier S. 144.

316 AUSSME, DS II 1333, AOK 8 ( Nr. 016/172 - gez. Italo Gariboldi) an das Oberkommando der Heeresgruppe B vom 6.1.1943; Hervorhebung im Original.

317 Revelli, Mai tardi, S. 201, Eintrag vom 9. 3. 1943.

318 Tolloy, Armata italiana, S. 92.

319 Dieser Begriff findet sich bei Ascari, Lunga marcia degli alpini, S. 73, der jedoch hinzufügte, daß dies bei der Bevölkerung weder zu Groll noch zu Feindseligkeit gegenüber den Italienern geführt habe. 
bemühten, nachdem die Reste der ARMIR im Auffrischungsraum um Gomel angekommen waren ${ }^{320}$, doch mit den Ereignissen des Winters 1942/43 verstärkte sich eine achsenkritische, wenig germanophile Grundströmung, die es von Anfang an gegeben hatte und die nun immer deutlicher hervortrat.

Wie ein Lauffeuer verbreiteten sich Berichte, die davon zeugten, $\mathrm{da} ß$ sich die so mächtigen und gut ausgerüsteten Deutschen geweigert hätten, ihren italienischen Kameraden in Not beizustehen, während die Zivilbevölkerung selbstlos das wenige mit den Italienern geteilt hätte, das ihr noch geblieben sei ${ }^{321}$. Ob diese Berichte auf wahren Begebenheiten beruhten, ob sie die Realität verzerrten oder übertrieben wiedergegeben wurden, ob sie gar erfunden wurden, um den Verlust von Handfeuerwaffen und Ausrüstungsgegenständen zu erklären ${ }^{322}$, war dabei zweitrangig. Daß sich auch die deutschen Soldaten zumeist auf Schusters Rappen hatten nach Westen durchschlagen müssen - die 385. Infanteriedivision hatte von über $700 \mathrm{Kraftfahr-}$ zeugen keine 20 retten können ${ }^{323}$ - und nicht bequem in gepanzerten Halbkettenfahrzeugen (wie es die Legende will), wurde ebenso rasch verdrängt wie die Tatsache, daß es während der Rückzugskämpfe auch Akte kameradschaftlicher Solidarität zwischen Deutschen und Italienern sowie Auseinandersetzungen zwischen italienischen Soldaten ${ }^{324}$ gegeben hatte - von Übergriffen italienischer gegen deutsche Soldaten ganz zu schweigen ${ }^{325}$.

Denn wie es zu den vielfach beklagten Übergriffen und Gewalttaten von deutschen gegen italienische Soldaten gekommen ist, so sind diese auch im umgekehrten Fall bezeugt. Bei Hans Wimpffen ist etwa nachzulesen, ein Offizier des königlichen Heeres habe während des Rückzugs das Feuer aus seinen Geschützen eröffnen lassen, um eine deutsche Kolonne dazu zu zwingen, die Straße freizumachen ${ }^{326}$, und Oberst Heidkämper berichtete, daß ein italienischer Hauptmann damit gedroht habe, das Generalkommando des XXIV. Panzerkorps mit einem Maschinengewehr aus seiner Unterkunft "herausschießen zu wollen “327. Auch aus italienischen Selbstzeugnissen wird deutlich, daß Soldaten des königlichen Heeres in Streitfällen mit deutschen Kameraden bereit waren, von der Waffe Gebrauch zu machen ${ }^{328}$. Während es die deutsche Führung jedoch nur ausnahmsweise für opportun hielt, solchen Zwischenfällen nachzugehen, legte man auf italienischer Seite großen Wert

320 Vgl. etwa Dok. 13 und Dok. 15.

321 Vgl. etwa Dok. 29, S. 263f. Zahlreiche Beispiele werden berichtet in: Bedeschi (Hrsg.), Fronte russo, Bd. 1, S. $511 \mathrm{ff} .$, S. 563-566, S. $579 \mathrm{ff}$.

322 In Dok. 24 fallen die vielen Berichte über den Raub von ansonsten als veraltet geschmähten Schußwaffen durch gut bewaffnete deutsche Soldaten auf, die diesen Verdacht aufkommen lassen. Der Verlust von Waffen und Ausrüstung mußte schließlich begründet werden.

${ }^{323}$ BA-MA, RH 26-385/42, Brief eines Angehörigen der 385. Infanteriedivision vom 13. 2. 1943.

324 Vgl. etwa Rigoni Stern, Alpini im russischen Schnee, S. 85 f. oder S. 100.

$325 \mathrm{Vgl}$. - über die hier publizierten Dokumente hinaus - die ausgewogene Darstellung bei Massignani, Alpini e tedeschi, S. 129-138; zur Überlagerung und Bearbeitung des Erlebten vgl. auch Ceva, Riflessioni, in: ders., Guerra mondiale, S. 279-282; in einer Aktennotiz vom 24. 2. 1943 über einen Truppenbesuch bei der 387. Infanteriedivision (BA-MA, RH 24-24/192) heißt es, die Soldaten seien "erregt “ über die "Rücksichtslosigkeit der Italiener".

$326 \mathrm{Vgl}$. Wimpffen, Zweite ungarische Armee, S. 280.

327 Vgl. Dok. 12, S. 152.

328 Vgl. etwa Lo Faso, 5 Mesi, S. 141 f., Ascari, Lunga marcia degli alpini, S. 73 f.; Corradi, Ritirata di Russia, S. $157 f$. 
darauf, die Übergriffe der deutschen Verbündeten sorgfältig zu dokumentieren ${ }^{329}$. Die bis heute spürbare einseitige Betonung der Opferrolle der italienischen Soldaten an der Ostfront hat hier eine ihrer Wurzeln. Doch wie dem auch sei: Die Katastrophe am Don war ein "Wendepunkt" in den deutsch-italienischen Beziehungen $^{330}$. Wie bei El Alamein zerbrach hier die "Achse“ schon Monate vor Mussolinis Sturz, und es ist alles andere als ein Zufall, daß die Geschehnisse an der Ostfront nach dem 8. September 1943 einen Ansatzpunkt für die Propagandaoffensive der postfaschistischen Kräfte Italiens bildete, die auf die ausschnitthafte "Verkürzung" eines „viel umfassenderen Kriegserlebnisses “ und auf die Verdrängung der Erinnerung an den Krieg an der Seite der Deutschen hinauslief 331.

Neben den negativen Erfahrungen vieler Soldaten war es vor allem die quantitative Bilanz der Campagna di Russia, die dem Fundament der deutsch-italienischen Allianz einen schweren Schlag versetzte. Als die Trümmer der 8. Armee Anfang März 1943 den Raum Gomel - Neschin - Schlobin erreicht hatten, der ihr von der deutschen Führung zur Neuordnung zugewiesen worden war, begann das ganze Ausmaß der Katastrophe sichtbar zu werden. Die ARMIR hatte 97 Prozent ihrer Artillerie verloren ${ }^{332}$, 80 Prozent aller Reit-, Last- oder Zugtiere sowie 70 Prozent der Kraftfahrzeuge. Angesichts der dürftigen italienischen Rüstungsproduktion konnten diese Verluste kaum ersetzt werden, zumal das königliche Heer praktisch gleichzeitig in Nordafrika große Mengen an Waffen und Kriegsmaterial eingebüßt hatte. Ungeheuer waren auch die Ausfälle unter den Soldaten. Von den rund 230000 Angehörigen der Armee, so nimmt man heute an, waren zwischen Dezember 1942 und Februar 1943 rund 95000 Mann zu Tode gekommen oder in Gefangenschaft geraten; die Zahl der Verwundeten, Kranken oder an Erfrierungen Leidenden ging in die Zehntausende ${ }^{333}$. Allerdings ist es aufgrund der unsicheren Quellenbasis schwierig, genaue Angaben zu machen, so daß bis heute viele Fragen offen geblieben sind. So läßt sich beispielsweise nur schätzen, wie viele italienische Soldaten im Kampf gefallen sind, wie viele auf dem Rückzug vor Kälte oder Erschöpfung umkamen und wie viele in Kriegsgefangenschaft geraten sind. Neuere Arbeiten gehen davon aus, daß rund 25000 Mann gefallen oder während des Rückzugs buchstäblich und im schlimmsten Sinne des Wortes auf der Strecke geblieben sind. Trifft dies zu, dann machte die Rote Armee im Winter 1942/43 etwa 70000 italienische Kriegsgefangene, die ein grausames Schicksal erwartete. Etwa 22000 erreichten nicht einmal die Lager, sondern fielen den Strapazen auf dem Marsch, den klimatischen Verhältnissen im russischen Winter, dem Hunger oder der Willkür der Wachmannschaften zum Opfer. Von denjenigen, die es bis in die Gefangenenlager schafften, kamen

$329 \mathrm{Vgl}$. Dok. 12 und Dok. 24.

330 Deakin, Brutale Freundschaft, S. 247.

331 Klinkhammer, Kriegserinnerung in Italien, in: Cornelißen/Klinkhammer/Schwentker (Hrsg.), Erinnerungskulturen, S. $336 \mathrm{f}$.

$332 \mathrm{Vgl}$. Operazioni delle unità italiane al fronte russo, S. $464 \mathrm{f}$.

333 Vgl. Rapporto sui prigionieri di guerra italiani in Russia, S. 20; Andrea Romano, Russia, campagna di, in: de Grazia/Luzzatto (Hrsg.), Dizionario del fascismo, Bd. 2, S. 567. Das einschlägige Werk des Heeresgeneralstabs (Operazioni delle unità italiane al fronte russo, S. 487) geht von rund $85000 \mathrm{im}$ Winter $1942 / 43$ Gefallenen und Vermißten sowie von knapp 30000 Verwundeten und von Erfrierungen Betroffenen aus. Zu den Schwierigkeiten bei der Ermittlung der Verluste vgl. an einem Fallbeispiel Vicentini, Perdite della Divisione alpina "Cuneense ${ }^{a}$. 
nochmals $38000 \mathrm{um}$; viele von ihnen waren so entkräftet, daß sie noch in den ersten Monaten des Jahres 1943 eine leichte Beute der Infektionskrankheiten wurden, die unter den Gefangenen grassierten. Letztlich waren es genau 10032 ehemalige Soldaten der ARMIR, die Italien wiedersehen sollten ${ }^{334}$.

Unter diesen Bedingungen war an einen weiteren Einsatz der 8. Armee an der Ostfront nicht zu denken. Nachdem das italienische Armeeoberkommando schon mit dem 31. Januar 1943 „aus der Führung in der Front ausgeschieden “ war ${ }^{335}$, wurden die zerschlagenen Verbände aus der Ukraine nach Weißrußland verlegt. Gariboldi und sein Stab, die Alpini, die Männer des XXXV. Armeekorps und die Soldaten der „Sforzesca“ kehrten im März in die Heimat zurück ${ }^{336}$. Das II. Armeekorps mit den Divisionen „Ravenna“ und „Cosseria“ sollte dagegen neu formiert werden und das faschistische Italien weiterhin an der Ostfront repräsentieren. Doch dieses Projekt wurde schnell ad acta gelegt, da sich die deutsche und die italienische Führung nicht darüber einigen konnten, wie das Korps ausgerüstet und eingesetzt werden sollte. Dies lag nicht zuletzt daran, daß man in Italien angesichts der kritischen militärischen Lage im Mittelmeerraum und der politischen Krise des Faschismus nicht mehr dazu bereit war, wie noch 1941/42 stärkere eigene Kräfte zu mobilisieren $^{337}$, und daß man es auf deutscher Seite für verfehlt hielt, wertvolle Ausrüstung an ein Heer zu liefern, dessen Unzuverlässigkeit erwiesen zu sein schien ${ }^{338}$. So war es eine logische Konsequenz, auch noch die restlichen italienischen Truppen in die Heimat zurückzuverlegen. Als das II. Armeekorps die Sowjetunion im April/ Mai 1943 verlassen hatte, bedeutete dies das unspektakuläre Ende der Intervention Mussolinis in den deutsch-sowjetischen Krieg.

334 Vgl. Giusti, Prigionieri, S. 90-98 und S. 225-228.

335 BA-MA, MFB4 41403, Bl. 1141, KTB des Deutschen Generals beim italienischen AOK 8, Fintrag vom 31. 1. 1943.

336 Vgl. Schreiber, Italiens Teilnahme, in: Förster (Hrsg.), Stalingrad, S. 278-281.

337 In Rom dachte die militärische Führung nach der Niederlage bei El Alamein und der Landung anglo-amerikanischer Truppen in Marokko schon Mitte November daran, entweder die Alpini oder die Divisionen des CSIR von der Ostfront abzuziehen, um die Heimatverteidigung zu stärken. Vgl. Cavallero, Diario, S. 575 f., Eintrag vom 17.11. 1942. Zu den Ende 1942 einsetzenden - ergebnislosen - italienischen Bemühungen, die deutschen Verbündeten im allgemeinen und Hitler im besonderen von der Notwendigkeit eines Strategiewechsels (unter Umständen verbunden mit einem Separatfrieden mit der Sowjetunion) und einer Konzentration der Kräfte auf den Krieg im Mittelmeerraum zu überzeugen, vgl. Förster, Stalingrad, S. 54 ff., und Deakin, Brutale Freundschaft, S. 129-135 und S. 200-260.

338 Vgl. Lagebesprechungen, S. 80 f. (Abendlage vom 4. 3. 1943). 
